\title{
A hyperbolic-type affine invariant curve flow
}

\author{
Weifeng Wo, Feiyao Ma and Changzheng Qu
}

\begin{abstract}
A new hyperbolic version of affine geometric flow is proposed, which is a family of plane curve flows whose acceleration is constant along affine normal direction. The equations satisfied by the graph and support function of the curve under this flow give rise to fully nonlinear hyperbolic equations. By reducing the flow to a single nonlinear hyperbolic equation, we obtain the existence for local solutions of the flow. Global existence is established by a method of LeFloch-Smoczyk for studying the hyperbolic mean curvature flow. The equations for both perimeter and area of closed curves under this flow are also obtained. Based on this, we show that for a closed curve, the solution of Cauchy problem of this flow blows up in finite time. Furthermore, some group-invariant solutions to this flow are discussed.
\end{abstract}

\section{Introduction}

This paper is devoted to studying the hyperbolic version of an affine invariant geometric flow. We consider the Cauchy problem of the hyperbolic affine invariant planar curve flow

$$
\left\{\begin{array}{l}
\gamma_{t t}=\mathcal{N}, \\
\gamma(p, 0)=\gamma_{0}(p), \\
\gamma_{t}(p, 0)=\gamma_{1}(p),
\end{array}\right.
$$

where $\mathcal{N}$ is the affine normal, which is related to the Euclidean unit normal $\mathbf{n}$ and tangent vector $\mathbf{t}$ via $\mathcal{N}=k^{1 / 3} \mathbf{n}-\frac{1}{3} k^{-5 / 3} k_{s} \mathbf{t}, k$ is Euclidean curvature, and $s$ is the Euclidean arc-length.

The parabolic-type curve flows in Euclidean geometry have been studied extensively in the past decades. A large number of results about local and global existence, formation of singularities, long-time behavior, stability of solutions and applications in crystal growth and computer vision of curve flows have been obtained. See for examples $[2,6,11,13,18,33]$ and references 
therein. In particular, there are many interesting works (see $[6,12,14]$ and references therein) which consider the Euclidean curve-shortening problem

$$
\left\{\begin{array}{l}
\gamma_{t}=\gamma_{s s}=k \mathbf{n}, \\
\gamma(p, 0)=\gamma_{0}(p),
\end{array}\right.
$$

where $\gamma(p, t) \in \mathbb{R}^{2}$ is a family of closed curves and $s$ is the Euclidean arclength parameter of the curve. In an intriguing paper [12], Gage and Hamilton proved that a convex embedded plane curve remains convex and shrinks to a point in a finite time, becoming round in the process. Moreover, Grayson $[14,15]$ proved that a closed embedded plane curve remains embedded and eventually becomes convex. So the problem (1.2) in this case is wellunderstood. In the sequel, many works have been done on the generalized curve-shortening problems. One may refer to the book [6] and references therein for the details. When we look for flows which preserve length and area, some integrable systems were obtained in a natural way [7]. Since the affine curve-shortening flow has important applications in image processing and computer vision [1], it is of great interest to study the affine invariant geometric flows. In [29], Sapiro and Tannenbaum introduced the affine analog of the Euclidean curve-shortening flow

$$
\left\{\begin{array}{l}
\gamma_{t}=\mathcal{N}=\gamma_{\rho \rho}, \\
\gamma(p, 0)=\gamma_{0}(p),
\end{array}\right.
$$

where $\mathcal{N}$ is the affine normal and $\rho$ is the affine arc-length parameter. They obtained evolution equations for the affine curvature and other affine invariants. As a result, the affine isoperimetric inequality was proved. Some properties of this flow were also explored [29]. Furthermore, Angenent et al. [5] verified that any smooth embedded curve converges to a point. This is analogous to the result on the Euclidean curve-shortening problem. An affineinvariant evolution equation for convex hypersurfaces driven by its affine normal in Euclidean space was studied in Andrews [3]. In [4], Andrews discussed the global existence and long-time behavior of the affine invariant fourth-order flow

$$
\left\{\begin{array}{l}
\gamma_{t}=\mu \mathcal{N}, \\
\gamma(p, 0)=\gamma_{0}(p),
\end{array}\right.
$$

where $\mu$ is the affine curvature of the curve $\gamma(p, t)$. He proved that the flow expands to infinity and tends to an ellipse after suitable normalization. 
A natural question arises: what are the hyperbolic versions of the curveshortening flows (1.2) and (1.3).

In Yau [32], it was proposed to study the motion of a hypersurface whose acceleration is equal to its mean curvature along the normal direction. In $\mathrm{He}$ et al. [17], local solvability of the following hyperbolic mean curvature flow:

$$
\frac{\partial^{2} X}{\partial t^{2}}=H \mathbf{n}
$$

for hypersurface $X(p, t)$ was established, and formation of singularities in finite time and asymptotic behavior of the flow were also examined. The hyperbolic version of the curve-shortening problem was studied by Kong and Wang [20] where several criteria on finite time blow-up for graphs were obtained. In Kong et al. [21], they also studied the hyperbolic curveshortening problem for convex curves. In LeFloch and Smoczyk [22], the hyperbolic mean curvature flow

$$
\left\{\begin{array}{l}
\frac{\partial^{2} X}{\partial t^{2}}=e H \mathbf{n}-\nabla \mathbf{e} \\
\left(\frac{\partial X}{\partial t}\right)_{t=0}^{T}=0 \\
X(p, 0)=X_{0}
\end{array}\right.
$$

was introduced and studied, where $H$ is the mean curvature of the hypersurface $X(p, t)$ and $e=\frac{1}{2}\left(\left|\frac{d X}{d t}\right|^{2}+n\right)$ is the local energy density. They established local well-posedness and uniqueness of classical solutions within the class of weak solutions. Some criteria that ensure the blow-up of the flow due to the formation of geometric singularities were provided. In Chou and Wo [9], the hyperbolic Gauss curvature flow for convex hypersurfaces was proposed. The local solvability of the flow under certain conditions was established. They also studied blow up and asymptotic behavior of the solutions. Other hyperbolic-type geometric flows were considered in [16, 28, 31].

One may compare (1.1) with (1.3), their difference may be explained by the structure of the flows. If we look at the evolution equations for the graph of a curve, (1.3) is equivalent to a second-order parabolic equation and (1.1) is a second-order hyperbolic equation. Therefore, the flow (1.1) can be regarded as the hyperbolic version of the affine curve-shortening flow (1.3).

The aim of this paper is to study the hyperbolic affine invariant curve flow (1.1), whose acceleration is constant along the affine normal direction. Interestingly, we find that the equation of the curve flow (1.1) is reduced to 
a single fully nonlinear hyperbolic equation

$$
u_{t t}=\frac{u_{x t}^{2}}{u_{x x}}+u_{x x}^{\frac{1}{3}},
$$

where $(x, u(x, t))$ is the graph of curve $\gamma$, or

$$
h_{t t}=-\left(\frac{1}{h_{\theta \theta}+h}\right)^{\frac{1}{3}}
$$

where $h(\theta, t)$ is the support function of $\gamma$. Instead of the flow (1.1), we shall study the local solvability and finite time blow-up of Equation (1.5) or (1.6). On such fully nonlinear hyperbolic-type equation, there is no well-developed theory. The equation under consideration has some nice invariant properties, not shared by other fully nonlinear hyperbolic-type equations, for instance it has a large group of symmetries. For the Euclidean and affine curveshortening flows, group-invariant solutions play crucial roles in the study of asymptotical behavior and singularities. Since Equation (1.5) is invariant under the affine transformation, we are also interested in group-invariant solutions of Equation (1.5).

The approach used in classifying the affine invariant hyperbolic equations is based on the group invariant theory of partial differential equations, which can be found in Olver [23]. The local existence of the flow is proved based on the standard theory from Taylor [30]. The global existence is proved by utilizing the method in LeFloch and Smoczyk [22] on the hyperbolic mean curvature flows (see also [19]). Note that the affine invariant hyperbolic equation admits rich symmetries. Based on the symmetry group theory on differential equations [23], we are able to obtain the corresponding groupinvariant solutions.

The outline of this paper is as follows. In Section 2, we first classify all affine invariant second-order hyperbolic equations, the hyperbolic-type affine invariant geometric flow (1.1) corresponding to a hyperbolic equation is obtained. Then evolution equations for other geometric quantities including graph and the support function of the curve associated to the flow are obtained. Furthermore, affine invariant property of the equation is studied. In Section 3, we prove the local existence of the flow and establish the global existence of solutions with bounded variation. In Section 4, we show that the evolving curve governed by this flow remains convex as long as it exists. Finite time blow-up and asymptotic behavior for closed curves driven by this flow are studied. Finally in Section 5, we study some group-invariant solutions of this flow. 


\section{The evolution equations}

We begin our investigation by classifying all second-order hyperbolic equations which are invariant under the linear affine group. Before proceeding further, we assume that for motions depending only on the geometry of the curves, the motion law is geometric. In particular, any solutions of affine invariant geometric flows are preserved under any reparametrization and the affine transformation. It turns out that the flow is geometric only if the corresponding equation for the graph is affine invariant. In case under the affine motion $\mathcal{R},(y, v)=\mathcal{R}(x, u)$, the graphs $(x, u(x, t))$ go over to graphs $(y, v(y, t))$, then $v(y, t)$ satisfies the same equation with $x$ and $u$ replaced by $y$ and $v$, respectively. More details can be found in Olver et al. [23-26] for discussions on group-invariant differential equations and their classifications.

Recall that the affine group acting linearly on $(x, u)$ and trivially on $t$ is a subgroup of the general linear group $s l(3)$, its infinitesimal symmetries are spanned by

$$
A(2)=\left\{\partial_{x}, \partial_{u}, u \partial_{x}, x \partial_{u}, x \partial_{x}-u \partial_{u}\right\}
$$

According to Lie's theory for symmetries, the fully nonlinear hyperbolic equation of the form

$$
u_{t t}=F\left(x, u, u_{x}, u_{t}, u_{x t}, u_{x x}\right)
$$

is affine invariant if and only if

$$
p r^{(2)} \mathbf{v}\left[u_{t t}-F\left(x, u, u_{x}, u_{t}, u_{x t}, u_{x x}\right)\right]=0
$$

holds on the solution manifold of $u_{t t}=F\left(x, u, u_{x}, u_{t}, u_{x t}, u_{x x}\right)$, where $\mathbf{v}$ is any vector in (2.1), and $p r^{(2)} \mathbf{v}$ is the second-order prolongation of $\mathbf{v}$ [23]. For any vector field of the form

$$
X=\tau(t, x, u) \frac{\partial}{\partial t}+\xi(t, x, u) \frac{\partial}{\partial x}+\phi(t, x, u) \frac{\partial}{\partial u}
$$

its second prolongation is defined by

$$
p r^{(2)} X=X+\phi^{t} \frac{\partial}{\partial u_{t}}+\phi^{x} \frac{\partial}{\partial u_{x}}+\phi^{t t} \frac{\partial}{\partial u_{t t}}+\phi^{t x} \frac{\partial}{\partial u_{t x}}+\phi^{x x} \frac{\partial}{\partial u_{x x}}
$$


where

$$
\begin{aligned}
\phi^{x} & =D_{x} \phi-u_{x} D_{x} \xi-u_{t} D_{x} \tau, \\
\phi^{t} & =D_{t} \phi-u_{x} D_{t} \xi-u_{t} D_{t} \tau, \\
\phi^{x x} & =D_{x} \phi^{x}-u_{x x} D_{x} \xi-u_{x t} D_{x} \tau, \\
\phi^{x t} & =D_{t} \phi^{x}-u_{x x} D_{t} \xi-u_{x t} D_{t} \tau, \\
\phi^{t t} & =D_{t} \phi^{t}-u_{t x} D_{t} \xi-u_{t t} D_{t} \tau .
\end{aligned}
$$

Here, $D_{x}$ and $D_{t}$ denote the operators of total differentiation with respect to $x$ and $t$, respectively. We first have the following result.

Theorem 2.1. Any affine invariant evolution equations $u_{t t}=F\left(x, u, u_{x}, u_{t}\right.$, $\left.u_{x t}, u_{x x}\right)$ possess the form

$$
u_{t t}=\frac{u_{x t}^{2}}{u_{x x}}+u_{t} \Phi\left(\frac{u_{x x}}{u_{t}^{3}}\right),
$$

where $\Phi$ is an arbitrary function of the indicated variable.

Proof. By the prolongation formula [23], $p r^{(2)} \partial_{x}=\partial_{x}$, and so

$$
\operatorname{pr}^{(2)}\left(u_{t t}-F\left(x, u, u_{x}, u_{t}, u_{x x}, u_{x t}\right)\right)=-F_{x}=0,
$$

which implies that $F$ is independent of $x$. Similarly, we have $p r^{(2)} \partial_{u}=\partial_{u}$, it implies that $F$ is also independent of $u$. Now for $x \partial_{u}$, the second prolongation is given by $p r^{(2)} x \partial_{u}=x \partial_{u}+\partial_{u_{x}}$. Its action on $u_{t t}=F$ gives $F_{u_{x}}=0$, this tells us that $F$ is independent of $u_{x}$. Furthermore, by a direct computation, we have

$$
\begin{aligned}
p r^{(2)}\left(u \partial_{x}\right)= & u \partial_{x}-u_{x}^{2} \partial_{u_{x}}-u_{x} u_{t} \partial_{u_{t}}-3 u_{x} u_{x x} \partial_{u_{x x}} \\
& -\left(2 u_{x} u_{x t}+u_{t} u_{x x}\right) \partial_{u_{x t}}-\left(u_{t t} u_{x}+2 u_{t} u_{x t}\right) \partial_{u_{t t}}, \\
p r^{(2)}\left(x \partial_{x}-u \partial_{u}\right)= & x \partial_{x}-u \partial_{u}-2 u_{x} \partial_{u_{x}}-u_{t} \partial_{u_{t}}-3 u_{x x} \partial_{u_{x x}} \\
& -2 u_{x t} \partial_{u_{x t}}-u_{t t} \partial_{u_{t t}}
\end{aligned}
$$

The action of the above two vector fields on Equation (2.2) leads to

$$
\begin{aligned}
& u_{x} u_{t} F_{u_{t}}+3 u_{x} u_{x x} F_{u_{x x}}+\left(2 u_{x} u_{x t}+u_{t} u_{x x}\right) F_{u_{x t}}-\left(u_{t t} u_{x}+2 u_{t} u_{x t}\right)=0, \\
& u_{t} F_{u_{t}}+3 u_{x x} F_{u_{x x}}+2 u_{x t} F_{u_{x t}}-u_{t t}=0
\end{aligned}
$$


Inserting $u_{t t}=F$ into the above two equations, they are reduced to equations

$$
u_{x x} F_{u_{x t}}-2 u_{x t}=0
$$

and

$$
u_{t} F_{u_{t}}+3 u_{x x} F_{u_{x x}}+2 u_{x t} F_{u_{x t}}-F=0
$$

The first equation is readily solved to yield

$$
F=\frac{u_{x t}^{2}}{u_{x x}}+\Psi\left(u_{x x}, u_{t}\right)
$$

for some function $\Psi$. Plugging this into the second equation gives

$$
u_{t} \Psi_{u_{t}}+3 u_{x x} \Psi_{u_{x x}}-\Psi=0
$$

which can be solved to get $\Psi=u_{t} \Phi\left(\frac{u_{x x}}{u_{t}^{3}}\right)$ for some function $\Phi$. Therefore, $F\left(x, u, u_{x}, u_{t}, u_{x t}, u_{x x}\right)$ must be the form of $\frac{u_{x t}^{2}}{u_{x x}}+u_{t} \Phi\left(\frac{u_{x x}}{u_{t}^{3}}\right)$. This completes the proof of the theorem.

In particular, taking $\Phi(z)=z^{\frac{1}{3}}$ in Theorem 2.1, we obtain the simplest affine invariant hyperbolic Equation (1.5). Comparing (1.5) with the hyperbolic geometric flow, we find that it can be derived from the flow (1.1). We consider the case of graphs for the flow. Let us denote $\gamma(\cdot, t)$ as an entire graph $(x, u(x, t))$ locally. Taking inner product flow (1.1) with $\mathbf{n}=\left(-u_{x}, 1\right) / \sqrt{1+u_{x}^{2}}$ and $\mathbf{t}=\left(1, u_{x}\right) / \sqrt{1+u_{x}^{2}}$, respectively, we find that the equation in (1.1) splits into two equations, namely,

$$
u_{t t}+2 u_{x t} x_{t}+u_{x x} x_{t}^{2}=u_{x x}^{\frac{1}{3}}
$$

and

$$
x_{t t}=-\frac{1}{3} u_{x x}^{-\frac{5}{3}} u_{x x x}
$$

Setting

$$
x_{t}=-\frac{u_{x t}}{u_{x x}}
$$


Equation (2.4) is reduced to Equation (1.5). In fact, once we have a solution $u(x, t)$ of (1.5), differentiating Equation (2.6) with respect to $t$, we get

$$
\begin{aligned}
x_{t t} & =-\frac{u_{x t t}}{u_{x x}}+\frac{2 u_{x x t} u_{x t}}{u_{x x}^{2}}-\frac{u_{x x x} u_{x t}^{2}}{u_{x x}^{2}} \\
& =-\frac{1}{u_{x x}}\left(\frac{u_{x t}^{2}}{u_{x x}}+u_{x x}^{\frac{1}{3}}\right)_{x}+\frac{2 u_{x x t} u_{x t}}{u_{x x}^{2}}-\frac{u_{x x x} u_{x t}^{2}}{u_{x x}^{2}} \\
& =-\frac{1}{3} u_{x x}^{-\frac{5}{3}} u_{x x x}
\end{aligned}
$$

which means that Equations (2.5) and (2.6) are compatible. It implies that the affine invariant curve flow (1.1) is equivalent to (1.5) under the constraint (2.6) in the graph case. To study the motions of convex curves, it is convenient to express the flow in terms of the support function $h(\theta, t)$. Let $\theta \in[0,2 \pi)$ be the the normal angle of the curve, then

$$
\mathbf{n}=-(\cos \theta, \sin \theta), \quad \mathbf{t}=(-\sin \theta, \cos \theta)
$$

and the support function is a function of $\theta$ and time $t$, defined by

$$
h(\theta, t)=-\langle\gamma(p, t), \mathbf{n}\rangle,
$$

where $\gamma(p, t)$ is the point on the curve whose normal angle is $\theta$.

Proposition 2.1. Let $\gamma(\cdot, t)$ be a family of uniformly convex curves satisfying (1.1). Then it is normal preserving, that is for any $t>0$

$$
\frac{d \boldsymbol{n}}{d t}=0
$$

if and only if $\frac{d n}{d t}=0$ at $t=0$.

Proof. In view of $\mathcal{N}=k^{1 / 3} \mathbf{n}-\frac{1}{3} k^{-5 / 3} k_{s} \mathbf{t}$, we find $\left\langle\mathbf{n}, \mathcal{N}_{p}\right\rangle=0$. A direct computation shows

$$
\begin{aligned}
\frac{\partial}{\partial t}\left\langle\frac{d \mathbf{n}}{d t}, \gamma_{p}\right\rangle & =-\frac{\partial}{\partial t}\left\langle\mathbf{n}, \gamma_{p t}\right\rangle=-\left\langle\mathbf{n}_{t}, \gamma_{p t}\right\rangle-\left\langle\mathbf{n}, \gamma_{t t p}\right\rangle \\
& =-\left\langle\left\langle\mathbf{n}_{t}, \mathbf{n}\right\rangle \mathbf{n}+\frac{\left\langle\mathbf{n}_{t}, \gamma_{p}\right\rangle}{\left\langle\gamma_{p}, \gamma_{p}\right\rangle} \gamma_{p}, \gamma_{p t}\right\rangle \\
& =-\frac{\left\langle\gamma_{p}, \gamma_{p t}\right\rangle}{\left\langle\gamma_{p}, \gamma_{p}\right\rangle}\left\langle\frac{d \mathbf{n}}{d t}, \gamma_{p}\right\rangle
\end{aligned}
$$

It follows that if $\frac{d \mathbf{n}}{d t}=0$ at $t=0$, then $\frac{d \mathbf{n}}{d t}=0$ for all $t>0$. 
By Proposition 2.1, $\theta$ and $t$ are independent variables. So we have

$$
h_{t}=-\left\langle\gamma_{t}, \mathbf{n}\right\rangle
$$

and

$$
\frac{d\left\langle\gamma_{t}, \mathbf{n}\right\rangle}{d t}=\left\langle\gamma_{t t}, \mathbf{n}\right\rangle=k^{\frac{1}{3}}
$$

Hence, the support function evolves according to Equation (1.6).

Next, we derive the evolution equations for Euclidean curvature and affine curvature of the curves. For any convex curve, its Euclidean curvature can be represented by $k=1 /\left(h_{\theta \theta}+h\right)$. By a direct computation, one gets

$$
k_{t}=-\frac{h_{\theta \theta t}+h_{t}}{\left(h_{\theta \theta}+h\right)^{2}}
$$

and

$$
k_{t t}=2 \frac{\left(h_{\theta \theta t}+h_{t}\right)^{2}}{\left(h_{\theta \theta}+h\right)^{3}}-\frac{h_{\theta \theta t t}+h_{t t}}{\left(h_{\theta \theta}+h\right)^{2}}
$$

Hence, the curvature evolves according to the equation

$$
k_{t t}=k^{-1} k_{t}^{2}+k^{2}\left(\left(k^{\frac{1}{3}}\right)_{\theta \theta}+k^{\frac{1}{3}}\right) \text {. }
$$

The affine arc-length $d \rho$ and curvature $\mu$ are given in terms of the Euclidean arc-length and curvature by

$$
d \rho=k^{\frac{1}{3}} d s
$$

and

$$
\mu=k^{\frac{4}{3}}+\frac{1}{3}\left(k^{-\frac{5}{3}} k_{s}\right)_{s}
$$

By a direct computation, we deduce that the evolution equations for the Euclidean curvature and affine curvature

$$
k_{t t}=2 k^{-1} k_{t}^{2}+k \mu
$$

and

$$
\mu_{t t}=\frac{1}{3} \mu_{\rho \rho}+f\left(\mu_{\rho}, \mu_{t}\right)
$$

where $f$ is a function of the lower-order terms of $\mu$. 
The following propositions will be useful for further discussion.

Proposition 2.2. The perimeter $L(t)$ for any closed curve $\gamma(\cdot, t)$ of problem (1.1) satisfies

$$
\frac{d L(t)}{d t}=\int_{0}^{2 \pi} h_{t} d \theta
$$

and

$$
\frac{d^{2} L(t)}{d t^{2}}=-\int_{0}^{2 \pi} k^{\frac{1}{3}} d \theta
$$

Proof. By the definition of the perimeter, we have

$$
L(t)=\int_{0}^{2 \pi}\left|\gamma_{\theta}\right| d \theta=\int_{0}^{2 \pi} k^{-1} d \theta
$$

A direct calculation yields

$$
\frac{d L(t)}{d t}=-\int_{0}^{2 \pi} k^{-2} k_{t} d \theta=\int_{0}^{2 \pi}\left(h_{\theta \theta t}+h_{t}\right) d \theta=\int_{0}^{2 \pi} h_{t} d \theta
$$

and

$$
\frac{d^{2} L(t)}{d t^{2}}=\int_{0}^{2 \pi} h_{t t} d \theta=-\int_{0}^{2 \pi} k^{\frac{1}{3}} d \theta
$$

Proposition 2.3. The area $A(t)$ enclosed by the closed curve $\gamma(t)$ satisfies

$$
\frac{d A(t)}{d t}=\int_{0}^{2 \pi} h_{t} k^{-1} d \theta
$$

and

$$
\frac{d^{2} A(t)}{d t^{2}}=\int_{0}^{2 \pi}\left(-k^{-\frac{2}{3}}+h_{t}^{2}-h_{\theta t}^{2}\right) d \theta
$$


Proof. By the definition of the area for a closed curve, we get

$$
A(t)=-\frac{1}{2} \int_{\gamma}\langle\gamma, \mathbf{n}\rangle d s=\frac{1}{2} \int_{0}^{2 \pi} h k^{-1} d \theta .
$$

After a direct calculation, we deduce that

$$
\begin{aligned}
\frac{d A(t)}{d t} & =\frac{1}{2} \int_{0}^{2 \pi}\left(h_{t} k^{-1}-h k^{-2} k_{t}\right) d \theta=\frac{1}{2} \int_{0}^{2 \pi}\left(h_{t} k^{-1}+h\left(h_{\theta \theta t}+h_{t}\right)\right) d \theta \\
& =\frac{1}{2} \int_{0}^{2 \pi}\left(h_{t} k^{-1}+h_{t}\left(h_{\theta \theta}+h\right)\right) d \theta=\int_{0}^{2 \pi} h_{t} k^{-1} d \theta .
\end{aligned}
$$

Further differentiation gives

$$
\begin{aligned}
\frac{d^{2} A(t)}{d t^{2}} & =\int_{0}^{2 \pi}\left(h_{t t} k^{-1}-h_{t} k^{-2} k_{t}\right) d \theta \\
& =\int_{0}^{2 \pi}\left(-k^{-\frac{2}{3}}+h_{t}\left(h_{\theta \theta t}+h_{t}\right)\right) d \theta=\int_{0}^{2 \pi}\left(-k^{-\frac{2}{3}}+h_{t}^{2}-h_{\theta t}^{2}\right) d \theta .
\end{aligned}
$$

Thus, the proposition is proved.

\section{Existence of the problem (1.1)}

In this section, we consider local and global existence of the flow (1.1) for certain initial values. Our first result is on local existence result of the Cauchy problem (1.1).

Theorem 3.1. (Local solvability for the flow (1.1)) Assume that the initial curve $\gamma_{0}(p)$ is convex and the initial velocity satisfies $\left\langle\partial \gamma_{t}(0) / \partial p, \boldsymbol{n}\right\rangle=0$, $\gamma_{0}(p) \in H^{k}\left(\mathbb{S}^{1}\right)$ and $\gamma_{1}(p) \in H^{k-1}\left(\mathbb{S}^{1}\right), k>5 / 2$. Then there exists a positive $T_{\max } \leq \infty$ such that (1.1) has a unique solution $\gamma(t)$ in $C\left(\left[0, T_{\max }\right)\right.$, $\left.H^{k}\left(\mathbb{S}^{1}\right)\right) \bigcap C^{1}\left(\left[0, T_{\max }\right), H^{k-1}\left(\mathbb{S}^{1}\right)\right)$. It is smooth provided $\gamma_{0}(p)$ and $\gamma_{1}(p)$ are smooth. Moreover, it is maximal in the sense that if $T_{\max }$ is finite, either the Euclidean curvature of $\gamma(t)$ tends to infinity or

$$
\|\gamma(t)\|_{C^{2}\left(\mathbb{S}^{1}\right)} \rightarrow \infty, \text { as } t \rightarrow T_{\max }
$$

Proof. To prove this theorem, we first consider the initial value problem of the equation for the graph $(x, u(x, t))$ of the curve. Since the initial curve $\gamma_{0}$ is a $H^{k}$-curve, we can find open intervals $J_{\alpha}, \alpha=1, \ldots, N$, in different coordinates and $H^{k}$-functions $u_{0}^{\alpha}$ defined over $J_{\alpha}$ such that $\gamma_{0}$ can be described 
as the union of the graphs $\left\{x, u_{0}^{\alpha}(x): x \in J_{\alpha}\right\}, \alpha=1, \ldots, N$. Assume $u^{\alpha}$ is the solution with initial value $u_{0}^{\alpha}$ for each $\alpha$. Note that $u(x, t) \equiv u^{\alpha}$ satisfies (1.5). Differentiating equation (1.5) with respect to $x$ yields the quasi-linear equation

$$
u_{x t t}=\frac{2 u_{x t}}{u_{x x}} u_{x x t}-\frac{u_{x t}^{2}}{u_{x x}} u_{x x x}+\frac{1}{3} u_{x x}^{\frac{2}{3}} u_{x x x} .
$$

Setting

$$
v^{0}=u, v^{1}=u_{x}
$$

we arrive at

$$
\left\{\begin{array}{l}
v_{t t}^{0}=\frac{\left(v_{t}^{1}\right)^{2}}{\left(v^{1}\right)_{x}}+\left(v_{x}^{1}\right)^{\frac{1}{3}} \\
v_{t t}^{1}=\frac{2 v_{t}^{1}}{v_{x}^{1}} v_{x t}^{1}-\frac{\left(v_{t}^{1}\right)^{2}}{\left(v_{x}^{1}\right)^{2}} v_{x x}^{1}+\frac{1}{3}\left(v_{x}^{1}\right)^{-\frac{2}{3}} v_{x x}^{1} .
\end{array}\right.
$$

This is a second-order quasi-linear system for $\left(v^{0}, v^{1}\right)$, which can be written as a first-order system. Indeed, letting

$$
\left(\begin{array}{c}
v^{0} \\
v^{1}
\end{array}\right) \rightarrow\left(\begin{array}{c}
v_{t}^{0} \\
v_{x}^{0} \\
v_{t}^{1} \\
v_{x}^{1}
\end{array}\right) \equiv\left(\begin{array}{l}
w^{0} \\
w^{1} \\
w^{2} \\
w^{3}
\end{array}\right)
$$

then (3.1) becomes

$$
\left\{\begin{array}{l}
w_{t}^{0}=\frac{w^{2}}{w^{3}}+\left(w^{3}\right)^{\frac{1}{3}} \\
w_{t}^{2}=\frac{2 w^{2}}{w^{3}} w_{x}^{2}-\frac{\left(w^{2}\right)^{2}}{\left(w^{3}\right)^{2}}\left(w^{3}\right)_{x}+\frac{1}{3}\left(w^{3}\right)^{-\frac{2}{3}} w_{x}^{3} \\
w_{t}^{1}=w_{x}^{0} \\
w_{t}^{3}=w_{x}^{2}
\end{array}\right.
$$

where the last two equations are compatible since

$$
v_{x t}^{0}=v_{t x}^{0}, \quad v_{x t}^{1}=v_{t x}^{1}
$$


Therefore, (3.2) can be rewritten in the form

$$
\left[\begin{array}{l}
w^{0} \\
w^{1} \\
w^{2} \\
w^{3}
\end{array}\right]_{t}=\left(\begin{array}{llll}
0 & 0 & 0 & 0 \\
0 & a & 0 & b \\
1 & 0 & 0 & 0 \\
0 & 1 & 0 & 0
\end{array}\right)\left[\begin{array}{c}
w^{0} \\
w^{1} \\
w^{2} \\
w^{3}
\end{array}\right]_{x}+[\text { l.o.t. }],
$$

where

$$
a=\frac{2 w^{2}}{w^{3}}, \quad b=-\frac{\left(w^{2}\right)^{2}}{\left(w^{3}\right)^{2}}+\frac{1}{3}\left(w^{3}\right)^{-\frac{2}{3}} .
$$

Let

$$
A=\left(\begin{array}{llll}
0 & 0 & 0 & 0 \\
1 & 0 & 0 & 0 \\
0 & 0 & a & b \\
0 & 0 & 1 & 0
\end{array}\right)
$$

and

$$
R=\left(\begin{array}{cccc}
1 & 0 & 0 & 0 \\
0 & 1 & 0 & 0 \\
0 & 0 & 1 & -\frac{a}{2} \\
0 & 0 & -\frac{a}{2} & b+\frac{a^{2}}{2}
\end{array}\right)
$$

Consequently, we have

$$
A^{\prime}=R A=\left(\begin{array}{cccc}
0 & 0 & 0 & 0 \\
0 & 0 & 0 & 0 \\
0 & 0 & -\frac{a}{2} & b \\
0 & 0 & b & -\frac{a b}{2},
\end{array}\right) .
$$

Note that $A^{\prime}$ is symmetric, and the system (3.3) becomes

$$
R \mathbf{w}_{t}=A^{\prime} \mathbf{w}_{x}+\text { l.o.t. }
$$

where l.o.t. denotes the lower-order terms. We can show that $R$ is positive definite. Indeed, a direct computation yields

$$
\langle\xi, R \xi\rangle=\xi_{1}^{2}+\xi_{3}^{2}+\xi_{2}^{2}-a \xi_{2} \xi_{4}+\left(b+\frac{a^{2}}{2}\right) \xi_{4}^{2} .
$$

It is easy to see that

$$
\Delta=a^{2}-4\left(b+a^{2} / 2\right)=-4\left(b-a^{2} / 2\right)=-\frac{1}{3}\left(w^{3}\right)^{-\frac{2}{3}}<0 .
$$


It follows that Equation (2.7) is symmetric hyperbolic. The standard result in [30] implies that for any $\mathbf{w}(0) \in H^{k}(\mathbb{R}), k>3 / 2, \mathbf{w}=\left\{w^{0}, w^{1}, w^{2}, w^{3}\right\}$ is locally solvable in $C\left([0, T), H^{k}(\mathbb{R})\right) \bigcap C^{1}\left([0, T), H^{k-1}(\mathbb{R})\right)$, and w is smooth if $\mathbf{w}(0)$ is smooth. Note that $w_{x}^{0}=w_{t}^{1}$, i.e., $\left(u_{t}\right)_{x}=\left(u_{x}\right)_{t}$. It is inferred from (2.7) that $\left(w^{0}\right)_{t x}=\left(w^{1}\right)_{t t}$, and $\left.w_{x}^{0}\right|_{t=0}=\left.w_{t}^{1}\right|_{t=0}=u_{x}^{1}$, hence $\left(u_{t}\right)_{x}=\left(u_{x}\right)_{t}$. Therefore $\gamma$ is locally solvable in $C\left([0, T), H^{k}\left(\mathbb{S}^{1}\right)\right) \bigcap C^{1}\left([0, T), H^{k-1}\left(\mathbb{S}^{1}\right)\right)$, $k>5 / 2$.

Furthermore, suppose $\|\mathbf{w}\|_{C^{1}}$ is uniformly bounded for $t \in[0, T)$, then there exists $T_{1}>T$ such that the solution can be extended to $C\left(\left[0, T_{1}\right)\right.$, $\left.H^{k}(\mathbb{R})\right)$. It follows that there exists a unique local solution to (1.1) on the maximal interval $\left[0, T_{\max }\right), T_{\max } \leq \infty$, in the sense that when $T_{\max }$ is finite, either the Euclidean curvature of $\gamma(t)$ tends to infinity, or

$$
\|\gamma(t)\|_{C^{2}\left(\mathbb{S}^{1}\right)} \rightarrow \infty, \text { as } \quad t \rightarrow T_{\max }
$$

In the following, we consider the case that $\gamma(p, t)$ satisfies (1.1) and each $\gamma(p, t)$ is an entire graph over $\mathbb{R}$. We assume that $\gamma(p)=(x(t), u(x, t))$ satisfies $(1.5)$ with $u(x, 0)=u_{0}(x)$ and $u_{t}(x, 0)=u_{1}(x), x \in \mathbb{R}$. The hyperbolictype affine geometric flow (1.1) reads

$$
\left\{\begin{array}{l}
u_{t t}=\frac{u_{x t}^{2}}{u_{x x}}+u_{x x}^{\frac{1}{3}}, \\
u(x, 0)=u_{0}(x), u_{t}(0, x)=u_{1}(x), x \in \mathbb{R},
\end{array}\right.
$$

which describes the vibrations of an infinitely long string, with initial position $u_{0}$ and initial velocity $u_{1}$.

Theorem 3.2. (Global solvability of the flow (1.1)) Assume that there exists a constant $\delta_{0}>0$ such that the initial data $u_{0}=u(x, 0)$ and $u_{1}=$ $u_{t}(x, 0): \mathbb{R} \rightarrow \mathbb{R}$ satisfy

$$
T V\left(u_{0 x x}\right)+T V\left(u_{1 x} / u_{0 x x}\right)<\delta_{0}
$$

where $T V$ is the total variation in $\mathbb{R}$. Then, the initial-value problem (1.1) in the form (3.5) has a global solution such that the functions $u_{x x}$ and $u_{x t} / u_{x x}$ have bounded variation in $\mathbb{R}$, uniformly in time.

Proof. Since

$$
\operatorname{det}\left(\begin{array}{cc}
-1 & \frac{u_{x t}}{u_{x x}} \\
\frac{u_{x t}}{u_{x x}} & -\frac{u_{x t}^{2}}{u_{x x}^{2}}+\frac{1}{3} u_{x x}^{-\frac{2}{3}}
\end{array}\right)=-\frac{1}{3} u_{x x}^{-\frac{2}{3}}<0
$$


It implies that the graph Equation (2.4) of the flow (1.1) is hyperbolic. Setting

$$
p=u_{x x}, \quad q=-\frac{u_{x t}}{u_{x x}},
$$

Equation (2.4) can be written in the conservation laws

$$
\left\{\begin{array}{l}
p_{t}+(p q)_{x}=0 \\
(p q)_{t}+\left(p q^{2}+p^{\frac{1}{3}}\right)_{x}=0
\end{array}\right.
$$

This is equivalent to the system

$$
\left(\begin{array}{l}
p \\
q
\end{array}\right)_{t}+A\left(\begin{array}{l}
p \\
q
\end{array}\right)_{x}=0
$$

with

$$
A=\left(\begin{array}{cc}
0 & 1 \\
\frac{1}{3} p^{-\frac{1}{3}}-\frac{q^{2}}{p^{2}} & \frac{2 q}{p}
\end{array}\right) .
$$

Clearly, $A$ has two eigenvalues given by

$$
\lambda_{ \pm}=\frac{3 q \pm \sqrt{3} p^{\frac{5}{6}}}{3 p}
$$

while the corresponding eigenvectors are

$$
\mu_{ \pm}=\left(\frac{3 p}{ \pm \sqrt{3} p^{\frac{5}{6}}+3 q}, 1\right)
$$

Regarded as a function of $p$ and $q$, the inner product between the gradient of $\lambda_{ \pm}$and $\mu_{ \pm}$equals

$$
\left\langle D \lambda_{ \pm}, \mu_{ \pm}\right\rangle=\frac{5}{6\left(p \pm \sqrt{3} p^{1 / 6} q\right)} \neq 0
$$

Hence, the hyperbolic system under consideration is genuinely nonlinear in the sense of Lax. Therefore, we can apply the global existence theorem in LeFloch and Smoczyk [22] to derive the global solution $(p, q)$ with bounded variation of initial values when the initial data have small bounded variation. 


\section{Finite time blow-up}

In this section, we deduce lower bound for the Euclidean curvature $k$ of the solution $\gamma$ of problem (1.1). Finite time blow-up for closed curves is also investigated.

Lemma 4.1. (preserving convexity) Let $k_{0}$ be the curvature of initial curve $\gamma_{0}$ and set $\delta=\min _{\theta \in[0,2 \pi]}\left\{k_{0}(\theta)\right\}$. Assume that $\delta>0$ and the initial velocity satisfies $k_{t}(0) \geq 0$. Then, for any $C^{2}$-solutions $k$ of $(2.7)$, one has

$$
k(\theta, t) \geq \delta
$$

for $t \in[0, T)$, where $T$ is the maximal existence time for the solution $\gamma$ of (1.1).

Proof. Note that the curvature satisfies (2.7), i.e.,

$$
\frac{1}{3} k^{\frac{4}{3}} k_{\theta \theta}-k_{t t}-\frac{2}{9} k^{\frac{1}{3}} k_{\theta}^{2}+k^{-1} k_{t}^{2}+k^{\frac{7}{3}}=0 .
$$

We can define the operator $L$ as follows:

$$
L[k] \equiv a k_{\theta \theta}+2 b k_{\theta t}+c k_{t t}+d k_{\theta}+e k_{t}+f k
$$

where $a=\frac{1}{3} k^{\frac{1}{3}}, b=0, c=-1, d=-\frac{2}{9} k^{\frac{1}{3}} k_{\theta}, e=k^{-1} k_{t}, f=k^{\frac{4}{3}} . a, b$ and $c$ are twice continuously differentiable, $d$ and $e$ are continuously differentiable. A direct computation shows

$$
b^{2}-a c=\frac{1}{9} k^{\frac{2}{3}}>0
$$

Hence, the operator $L$ defined by $(4.1)$ is hyperbolic in the domain $\mathbb{S}^{1} \times$ $[0, T)$.

Setting $\tilde{k}=-k+\delta$, it satisfies

$$
\begin{cases}L[\tilde{k}] \geq 0, & \text { in } \mathbb{S}^{1} \times\left[0, T_{0}\right), \\ \tilde{k}(\theta, 0) \leq 0, & \text { on } t=0, \\ \frac{\partial \tilde{k}}{\partial \nu}+\left(b_{\theta}+c_{t}-e\right) \tilde{k}=-\delta k^{-1}(0) k_{t}(0) \leq 0, & \text { on } t=0 .\end{cases}
$$


Applying the maximum principle for hyperbolic equations [27], we conclude that

$$
\tilde{k} \leq 0, \quad \text { i.e., } \quad \delta \leq k(\theta, t) \text { in } \mathbb{S}^{1} \times\left[0, T_{0}\right)
$$

with $T_{0} \leq T$, which means that the solution curve $\gamma$ is convex on $\mathbb{S}^{1} \times\left[0, T_{0}\right)$.

Theorem 4.1. Let the initial curve $\gamma_{0}$ be a smooth, strictly convex closed curve. Then, the solution of the flow (1.1) exists only at a finite-time interval $\left[0, T_{\max }\right)$. More precisely, one of the followings must be true:

(i) the solution of the flow converges to a point, i.e., the curvature of the curve blows up at finite time $T_{\max }$;

(ii) the curvature of the curve is discontinuous at $t=T_{\max }$, so the solution converges to a piecewise smooth curve.

Proof. By Proposition 2.2, for any closed curve we have

$$
\frac{d^{2} L(t)}{d t^{2}}=-\int_{0}^{2 \pi} k^{\frac{1}{3}} d \theta=-\int_{\gamma} k^{\frac{4}{3}} d s
$$

Using Cauchy-Schwartz inequality

$$
\begin{gathered}
\left(\int_{\gamma} k^{\frac{4}{3}} d s\right)^{\frac{3}{4}}\left(\int_{\gamma} d s\right)^{\frac{1}{4}} \geq \int_{\gamma} k d s=2 \pi \\
\frac{d^{2} L}{d t^{2}} \leq-\frac{(2 \pi)^{\frac{4}{3}}}{L^{\frac{1}{3}}}
\end{gathered}
$$

It follows that if $\frac{d}{d t} L\left(t_{0}\right) \leq 0$ at some instant $t_{0}$, then $\frac{d L}{d t}$ and $L$ will decrease for all $t \geq t_{0}$. Hence, we deduce that $\frac{d^{2} L}{d t^{2}} \leq-\frac{(2 \pi)^{\frac{4}{3}}}{L^{\frac{1}{3}}\left(t_{0}\right)}$ and $L$ becomes zero in finite time. On the other hand, when $\frac{d L}{d t}>0$ for all $t$, we have

$$
\frac{1}{2} \frac{d}{d t}\left(\frac{d L}{d t}\right)^{2} \leq-(2 \pi)^{\frac{4}{3}} \frac{d}{d t}\left(L^{\frac{2}{3}}\right)
$$

which gives

$$
\left(\frac{d L}{d t}\right)^{2}(t) \leq\left(\frac{d L}{d t}\right)^{2}\left(t_{0}\right)-2(2 \pi)^{\frac{4}{3}}\left(L^{\frac{2}{3}}(t)-L^{\frac{2}{3}}\left(t_{0}\right)\right)
$$


It implies that $L(t)$ cannot expand to infinity, and $\frac{d^{2} L(t)}{d t^{2}} \leq-\frac{(2 \pi)^{\frac{4}{3}}}{c_{0}}$, for some $c_{0}>0$. However, then

$$
\frac{d L}{d t}(t) \leq \frac{d L}{d t}\left(t_{0}\right)-\frac{(2 \pi)^{\frac{4}{3}}}{c_{0}}\left(t-t_{0}\right)
$$

contradiction holds as $t \rightarrow \infty$. We conclude that there exists a finite time $T_{0}$ such that $L\left(T_{0}\right)=0$.

Let $\left[0, T_{\max }\right)$ be the maximal time interval for the solution curve $\gamma$ with smooth closed initial curve. By Lemma 4.1, we know that $\gamma$ remains strictly convex on $\left[0, T_{\max }\right)$.

If $T_{0} \leq T_{\max }$, where $T_{\max }$ is the maximal existence time of the flow, then $T_{\max }$ must equal to $T_{0}$ by the definition of $T_{\max }$. Therefore, the solution curve converges to a point.

If $T_{0}>T_{\max }$, which means $L\left(T_{\max }\right)>0$. By Theorem 3.1, one has

$$
\|\gamma(t)\|_{C^{2}\left(S^{1}\right)} \rightarrow \infty, \text { as } t \rightarrow T_{\max }
$$

It follows that the Euclidean curvature $k$ must be discontinuous at $T_{\max }$.

\section{Group-invariant solutions}

Group-invariant solutions such as traveling waves, rotating waves, expanding and contracting self-similar solutions play important roles in the study of geometric flows, such as the curve-shortening problem, etc. $[6-8,10]$. To explore properties of general solutions of nonlinear evolution equations, we need to know some group-invariant solutions. These group-invariant solutions are often used to describe blow-up scenario and long-time behavior of more general solutions.

The reduced equation of flow (1.1) is a fully nonlinear hyperbolic equation. Special group-invariant solutions are helpful to understand its singularities. So, in this section, we attempt to study some group-invariant solutions and their long-time behavior.

To obtain group-invariant solutions of the flow (1.1), we need to write the flow (1.1) in terms of graph of the curves. Assume that the plane curve $\gamma(\cdot, t)$ is given locally as a graph of the form $(x, u(x, t))$, the flow (1.1) is converted to a nonlinear hyperbolic Equation (1.5). Since the equation is geometric, it must admit the affine motion as its symmetries. Furthermore, being independent of $t$ means that it admits translation in $t$. The special form of (1.5) suggests that it admits a certain scaling invariance. Making use 
of Lie's infinitesimal criterion for symmetry [23], after a routine computation we have the following result.

Proposition 5.1. The Lie algebra of all infinitesimal symmetries of (1.5) is spanned by

$$
\left\{\partial_{x}, \partial_{t}, \partial_{u}, u \partial_{x}, x \partial_{u}, x \partial_{x}-u \partial_{u}, x \partial_{x}+u \partial_{u}+\frac{2}{3} t \partial_{t}, t \partial_{x}, t \partial_{u}\right\} .
$$

The proof of this proposition is omitted here.

Note that Equations (1.5) and (1.6) are derived from the same curve flow, we can convert vector fields in Proposition (5.1) on the jet space $\left(x, t, u, u_{x}\right)$ to the jet space $\left(\theta, t, h, h_{\theta}\right)$. The support function of the curve $\gamma$ is represented by $h(\theta)=\langle\gamma(\theta),(\cos \theta, \sin \theta)\rangle$, where $\theta$ is the normal angle. We have $h_{\theta}=-x \sin \theta+u \cos \theta$. Therefore, there hold the following relations between graph and support function [8]

$$
h=\frac{u-x u_{x}}{\left(1+u_{x}^{2}\right)^{\frac{1}{2}}} \text { and } h_{\theta}=-\frac{x+u u_{x}}{\left(1+u_{x}^{2}\right)^{\frac{1}{2}}} .
$$

Therefore, $\partial_{x}, \partial_{u}$ and $\partial_{u_{x}}$ can be represented by $\partial_{h}$ and $\partial_{h_{\theta}}$. Finally, the vector field in the $(x, t, u)$-coordinates can be converted to new one in the $(\theta, t, h)$-coordinates. The following table shows the results of the conversion from $(x, t, u)$ to $(\theta, t, h)$.

According to the general theory for Lie symmetries, given any infinitesimal symmetry $v$, there usually corresponds a $v$-invariant solution. Two classes of affine-invariant solutions are discussed in some details in the following.

\subsection{Self-similar solutions}

A self-similar solution is a solution of (1.1) whose shapes change homothetically during the evolution. $\hat{\gamma}=\lambda(t) \gamma(\cdot)$ is a self-similar solution if and only if

$$
\lambda(t)^{\prime \prime} \lambda(t)^{\frac{1}{3}} \gamma \cdot \mathbf{n}=k^{\frac{1}{3}}
$$

Assume that this curve is not flat, then $\lambda(t)^{\prime \prime} \lambda(t)^{\frac{1}{3}}$ must be a non-zero constant. After a re-scaling, we may assume that the constant is $3 / 4$ or $-3 / 4$. On the other hand, we can get the constant $3 / 4$ from the point view of the group-invariant solution. In view of table 1 , the corresponding symmetry of the self-similar solution is $3 x \partial_{x}+3 u \partial_{u}+2 t \partial_{t}$, which is equivalent 
Table 1: Infinitesimal symmetries in $(x, t, u)$ and $(\theta, t, h)$.

\begin{tabular}{cc}
\hline$u_{t t}=\frac{u_{x t}^{2}}{u_{x x}}+\left(u_{x x}\right)^{\frac{1}{3}}$ & $h_{t t}=-\left(\frac{1}{h_{\theta \theta}+h}\right)^{\frac{1}{3}}$ \\
\hline$\partial_{x}$ & $\cos \theta \partial_{h}$ \\
$\partial_{u}$ & $\sin \theta \partial_{h}$ \\
$-u \partial_{x}+x \partial_{u}$ & $\partial_{\theta}$ \\
$\partial_{t}$ & $\partial_{t}$ \\
$3 x \partial_{x}+3 u \partial_{u}+2 t \partial_{t}$ & $2 t \partial_{t}+3 h \partial_{h}$ \\
\hline
\end{tabular}

to $2 t \partial_{t}+3 h \partial_{h}$, its group action is $(\theta, t, h) \rightarrow\left(\theta, e^{2 \epsilon} t, e^{3 \epsilon} h\right)$. Hence, the selfsimilar solution takes the form $t^{3 / 2} h(\theta)$, which satisfies $\lambda(t)^{\prime \prime} \lambda(t)^{\frac{1}{3}}=3 / 4$. Plugging this into Equation (1.6) yields

$$
\frac{3}{4} h=-\left(\frac{1}{h_{\theta \theta}+h}\right)^{\frac{1}{3}}
$$

If we plug $\lambda(t)^{\prime \prime} \lambda(t)^{\frac{1}{3}}=-3 / 4$ into Equation (4.2), the support function satifies

$$
\frac{3}{4} h=\left(\frac{1}{h_{\theta \theta}+h}\right)^{\frac{1}{3}} .
$$

The reduced Equations (5.1), and (5.2) are similar to the parabolic case [6]. The former one is called an expanding self-similar solution ( $\lambda$ expands to infinite) and the latter one is called a contracting self-similar solution $(\lambda$ tends to 0 at finite time).

For Equation (5.1), a typical solution subject to the initial conditions $h(0)=-\alpha, \alpha>0$ and $h_{\theta}(0)$ of (5.1) is an even, convex function, which is strictly increasing in $\left(0, \theta_{0}\right)$ where $\theta_{0}$ is the zero of $h$ and $h_{\theta}$ blows up as $\theta \uparrow \theta_{0}$. Then, the group-invariant solution determined by $h$ is a convex, complete noncompact curve lying inside the wedge $\left\{(x, y): y<|x| \tan \theta_{0}\right\}$ (see figure 1). Denote such curve by $\gamma_{0}(\theta)$. The group-invariant solution is the expanding self-similar solution $\gamma(\theta, t)=t^{3 / 2} \gamma_{0}(\theta)$.

Now, we move to (5.2). For convenience we choose $\lambda(t)^{\prime \prime} \lambda(t)^{\frac{1}{3}}$ to be -1 , instead of $-3 / 4$ by scaling. Then, the support function satisfies

$$
h=\left(\frac{1}{h_{\theta \theta}+h}\right)^{\frac{1}{3}},
$$




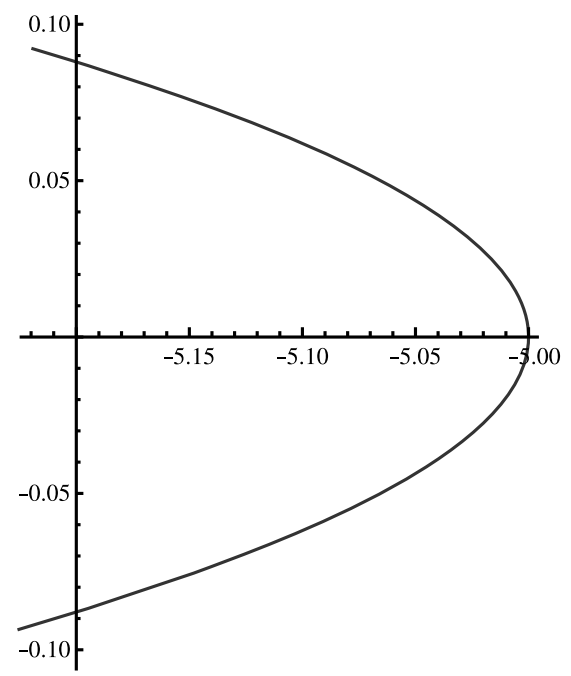

Figure 1: Expanding self-similar solution.

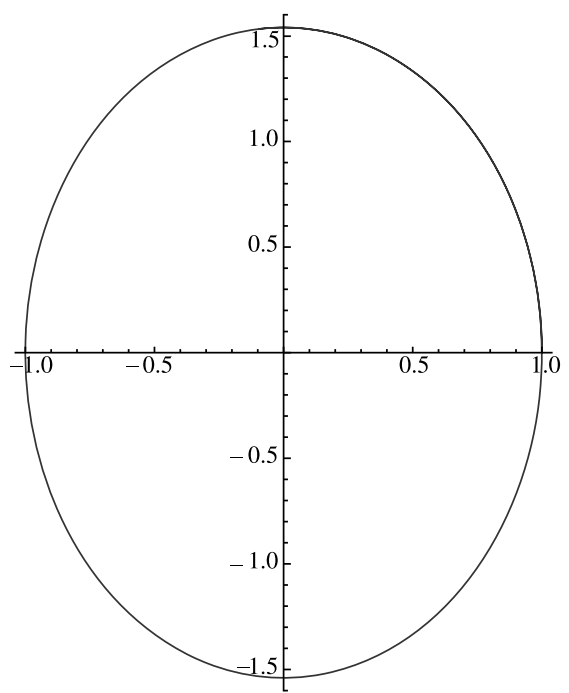

Figure 2: Contracting self-similar solution.

which can be solved explicitly for the initial conditions $h(0)=\alpha \geq 1, h_{\theta}(0)=$ 0 . All solutions of (5.3) are given by $\left[\alpha^{2} \cos ^{2} \theta+\alpha^{-2} \sin ^{2} \theta\right]^{1 / 2}$, which are the support functions of ellipses (see figure 2).

There is a special self-similar solution: circles, which can also be derived from the group generated by $\left\{u \partial_{x}-x \partial_{u}\right\}$. The corresponding group action 
is $(x, u, t) \rightarrow(x \cos \epsilon-u \sin \epsilon, u \cos \epsilon+x \sin \epsilon, t)$ and the group-invariants are $v=u^{2}+x^{2}$ and $y=t$. Thus, the corresponding group-invariant solution is given by

$$
u=\sqrt{v(y)-x^{2}},
$$

where $v(y)$ satisfies the equation

$$
2 v v_{y y}-v_{y}^{2}+4 v^{\frac{4}{3}}=0
$$

Set $v=r^{2}$, then (5.4) reduces to

$$
r^{\frac{1}{3}} r^{\prime \prime}+1=0
$$

It is easy to see that the group-invariant solution is a family of circles with radius $r$ and initial values $r(0)=r_{0}$ and $r^{\prime}(0)=r_{1}$. Multiplying Equation (5.5) by $r^{\prime} r^{1 / 3}$ and integrating the resulting equation, we get the following first-order ordinary differential equation:

$$
r^{\prime}=\left\{\begin{array}{l}
\sqrt{-3 r^{\frac{2}{3}}+r_{1}^{2}+3 r_{0}^{\frac{2}{3}}} \\
-\sqrt{-3 r^{\frac{2}{3}}+r_{1}^{2}+3 r_{0}^{\frac{2}{3}}}
\end{array}\right.
$$

It is easy to see $r^{\prime \prime}<0$, which means $r^{\prime}$ is a decreasing function and

(1) If the initial value $r^{\prime}(0)>0, r$ increases first, then decreases to 0 at finite time. The circles expand first, and then contract to a point in finite time.

(2) If the initial value $r^{\prime}(0)<0, r$ decreases to 0 at finite time. The circles contract to a point in finite time.

We show the graph of the radius $r(t)$ in the following figure for $r^{\prime}(0)>0$ and $r^{\prime}(0) \leq 0$. Figures 3 and 4 show that the radius of circles shrinks to 0 , which means circles are contracting self-similar solutions.

\subsection{Hyperbolic rotational invariant solutions}

In this subsection, we study the group-invariant solution corresponding to the hyperbolic rotational symmetry $\left\{u \partial_{x}+x \partial_{u}\right\}$. The group action is $(x, u, t) \rightarrow(x \cosh \epsilon+u \sinh \epsilon, u \cosh \epsilon+x \sinh \epsilon, t)$, and the group invariants 


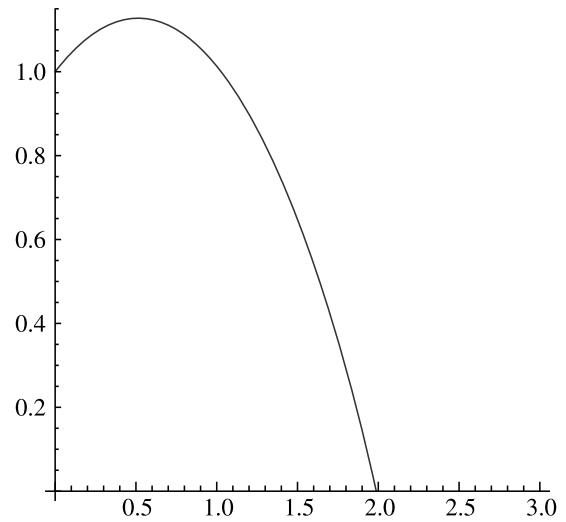

Figure 3: $r_{1}>0$.

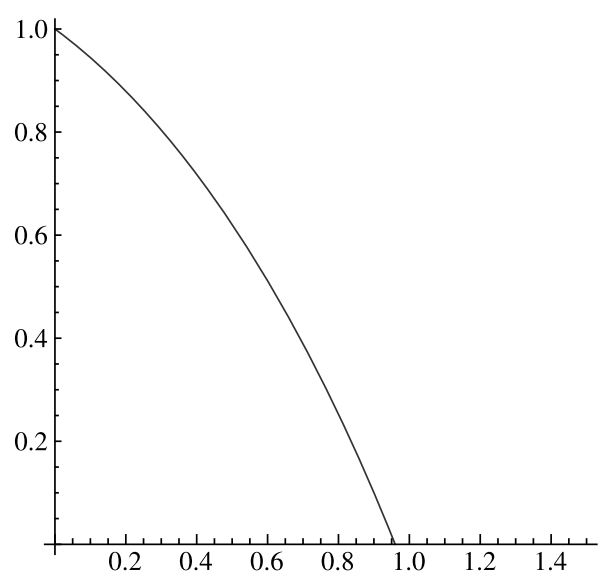

Figure 4: $r_{1} \leq 0$.

are $v=u^{2}-x^{2}$ and $y=t$. We have the corresponding group-invariant solution

$$
u=\sqrt{v(y)+x^{2}}
$$

where $v(y)$ satisfies

$$
2 v v_{y y}-v_{y}^{2}-4 v^{\frac{4}{3}}=0 .
$$

Multiplying Equation (5.6) by $v_{y} / v^{2}$ gives

$$
\left(\frac{v_{y}^{2}}{v}\right)^{\prime}=\left(12 v^{\frac{1}{3}}\right)^{\prime} .
$$


Integrating (5.7) from 0 to $y$ yields

$$
v_{y}^{2}=12 v^{\frac{4}{3}}+\left(\frac{v_{1}^{2}}{v_{0}}-12 v_{0}^{\frac{1}{3}}\right) v
$$

where $v(0)=v_{0}$ and $v_{y}(0)=v_{1}$. First, we consider solutions of (5.8) subject to the initial conditions $v(0)=a, a>0$ and $v_{y}(0)=0$. For each $a>0, v(y)$ is a convex function and monotonic. Since $v_{y}=\sqrt{12 v^{\frac{4}{3}}+12 v_{0}^{\frac{1}{3}}} v \leq C_{2} v^{\frac{2}{3}}$, when $v$ is large. Therefore $v^{\frac{1}{3}}<C_{2} y+C_{3}$, which means that $v$ expands to infinite, when $y$ goes to infinite, and $v$ does not blow up in finite time.

For solutions of (5.6) with the initial conditions $v(0)=-a, a>0$ and $v_{y}(0)=0$. It is ready to see that for each $a>0, v(y)$ is a concave function. Since $v_{y}=-\sqrt{12 v^{\frac{4}{3}}+12 v_{0}^{\frac{1}{3}} v}$, then $w(y)=-v(y)$ satisfies $w_{y}=$ $\sqrt{12 w^{\frac{4}{3}}+12 w_{0}^{\frac{1}{3}} w}$. Following the above discussion, we know that $w$ is strictly increasing and expands to infinite when $y$ goes to infinite. It implies that $v$ is strictly decreasing and expands to negative infinite when $y$ goes to infinite. Thus, the solution does not blow up in finite time.

\section{Acknowledgments}

The authors would like to thank Professor Kaiseng Chou for suggesting to consider the hyperbolic-type affine geometric flow. The work of Wo is supported by the NSF-China grant-11201249 and the Project Supported by Scientific Research Fund of Zhejiang Provincial Education Department Y201223456. The work of Ma is partially supported by Ningbo Natural Science Foundation (2012A610033). The work of Qu is supported in part by the NSF-China for Distinguished Young Scholars grant-10925104 and Ph.D Programs Foundation of Ministry of Education of China-20106101110008.

\section{References}

[1] L. Alvarez, F. Guichard, P.L. Lions and J.M. Morel, Axioms and fundamental equations of image processing, Arch. Ration. Mech. Anal., 123 (1993), 199-257.

[2] B. Andrews, Contraction of convex hypersurfaces in Euclidean space, Calc. Var. Partial Diff. Eq., 2 (1994), 151-171.

[3] B. Andrews, Contraction of convex hypersurfaces by their affine normal, J. Diff. Geom., 43 (1996), 207-230. 
[4] B. Andrews, The affine curve-lengthening flow, J. Reine Angew. Math., 506 (1999), 43-83.

[5] S. Angenent, G. Sapiro and A. Tannenbaum, On the affine heat equation for non-convex curves, J. Amer. Math. Soc., 11 (1998), 601-634.

[6] K.S. Chou and X.P. Zhu, The Curve Shortening Problem, Chapman \& Hall/CRC, Boca Raton, FL, 2001.

[7] K.S. Chou and C.Z. Qu, Integrable equations arising from motions of plane curves, Physica D, 162 (2002), 9-33.

[8] K.S. Chou and G.X. Li, Optimal systems and invariant solutions for the curve shortening problem, Commun. Anal. Geom., 10 (2002), 241-274.

[9] K.S. Chou and W.F. Wo, On hyperbolic Gauss curvature flows, J. Diff. Geom., 4 (2011), 455-486.

[10] Z.Z. Dong, Y. Chen, D.X. Kong and Z.G. Wang, Symmetry reduction and exact solutions of a hyperbolic Monge-Ampére equation, Chin. Ann. Math. Ser. B, 33 (2012), 309-316.

[11] K. Ecker, Regularity Theory for Mean Curvature Flow, Birkhäuser Boston, Inc., Boston, MA, 2004.

[12] M. Gage and R.S. Hamilton, The heat equation shrinking convex plane curves, J. Diff. Geom., 23 (1986), 69-96.

[13] Y. Giga, Surface Evolution Equations. A level set approach. Birkhäuser Verlag, Basel, 2006.

[14] M.A. Grayson, The heat equation shrinks embedded plane curves to round points, J. Diff. Geom., 26 (1987), 285-314.

[15] M.A. Grayson, Shortening embedded curves, Ann. Math., 129 (1989), $71-111$.

[16] M.E. Gurtin and P. Podio-Guidugli, A hyperbolic theory for the evolution of plane curves. SIAM J. Math. Anal., 22 (1991), 575-586.

[17] C.L. He, D.X. Kong and K.F. Liu, Hyperbolic mean curvature flow, J. Diff. Eq., 246 (2009), 373-390.

[18] G. Huisken and A. Polden, Geometric Evolution Equations for Hypersurfaces, 45-84, Lecture Notes in Mathematics, 1713, Springer, Berlin, 1999. 
[19] T. Iguchi and P.G. LeFloch, Existence theory for hyperbolic systems of conservation laws with general flux-functions, Arch. Ration. Mech. Anal., 168 (2003), 165-244.

[20] D.X. Kong and Z.G. Wang, Formation of singularities in the motion of plane curves under hyperbolic mean curvature flow, J. Diff. Equ., 247 (2009), 1694-1719.

[21] D.X. Kong, K.F. Liu and Z.G. Wang, Hyperbolic mean curvature flow: evolution of plane curves, Acta Math. Sci. B, 29B (2009), 493-514.

[22] P.G. LeFloch and K. Smoczyk, The hyperbolic mean curvature flow, J. Math. Pures Appl., 90 (2008), 591-614.

[23] P.J. Olver, Applications of Lie Groups to Differential Equations, Springer-Verlag, New York, 1998.

[24] P.J. Olver, Equivalence, Invariants, and Symmetry, Cambridge University Press, Cambridge, 1995.

[25] P.J. Olver, G. Sapiro and A. Tannenbaum, Differential invariant signatures and flows in computer vision: a symmetry group approach, in 'Geometry-Driven Diffusion in Computer Vision', ed. B.M. Ter Haar Romeny, Kluwer Academic Publishers, Dordrecht, 1994.

[26] R.H. Heredero and P.J. Olver, Classification of invariant wave equations, J. Math. Phys., 37 (1996), 6414-6438.

[27] M.P. Protter and H.F. Weinberger, Maximum Principles in Differential Equations, Prentice-Hall, New Jersey, 1967.

[28] H.G. Rotstein, S. Brandon and A. Novick-Cohen, Hyperbolic flow by mean curvature, J. Cryst. Growth, 198-199 (1999), 1256-1261.

[29] G. Sapiro and A. Tannenbaum, On affine plane curve evolution, J. Funct. Anal., 119 (1994), 79-120.

[30] M.E. Taylor, Partial differential equations III: nonlinear equations, Springer-Verlag, New York, 1996.

[31] T. Tsurumi, H. Segur, K. Nakayama and M. Wadati, Motion of curves specified by accelerations, Phys. Lett. A, 224 (1998), 253-263.

[32] S.T. Yau, Review of geometry and analysis, Asian J. Math., 4 (2000), $235-278$. 
[33] X.P. Zhu, Lectures on Mean Curvature Flows, American Mathematical Society, Providence, RI; International Press, Somerville, MA, 2002.

Department of Mathematics

NingBo University

NingBo 315211

People's Republic of China

Email address: woweifeng@nbu.edu.cn

Email address: mafeiyao@nbu.edu.cn

Email address: quchangzheng@nbu.edu.cn

RECEIVED JANUARY 28, 2013 
